\title{
BRAF-MEK inhibitor combo approved for adjuvant melanoma therapy
}

$\mathrm{O}$ n April 30, 2018, the US Food and Drug Administration expanded the indication for the combined use of dabrafenib and trametinib to include adjuvant treatment of $B R A F$-mutant melanoma following complete surgical resection. Dabrafenib is an inhibitor of the BRAF kinase, and trametinib is an inhibitor of the MEK kinase, both of which are components of the mitogen-activated protein kinase (MAPK) signaling pathway. The 2 drugs are already approved as both single agents and in combination for the treatment of $B R A F-$ mutated metastatic melanoma.

The current approval was based on data from a phase 3, international, multicenter, randomized, double-blind, placebo-controlled trial. The COMBI-AD trial was carried out from January 2013 through December 2014 at 169 sites in 26 countries. A total of 870 patients with stage III melanoma and BRAF V600E/K mutations and pathologic involvement of regional lymph nodes following complete resection were randomly assigned to receive dabrafenib 150 $\mathrm{mg}$ twice daily in combination with trametinib $2 \mathrm{mg}$ once daily, or 2 matched placebos for up to 1 year. Randomization was stratified according to $B R A F$ mutation status (V600E or V600K) and disease stage (IIIA, IIIB or IIIC).

Eligible patients were aged 18 years or older and had an Eastern Cooperative Oncology Group (ECOG) performance status of 0 or 1 (on a scale of 1-5, with higher scores indicating greater disability). Patients who had undergone previous systemic anticancer therapy or radiotherapy were excluded from the study.

The primary endpoint was relapse-free survival (RFS), defined as the time from randomization to disease recurrence or death from any cause. Secondary endpoints included overall survival (OS), distant metastasis-free survival (DMFS), freedom from relapse (FFR), and safety. Clinical examination and imaging by computed tomography, magnetic resonance imaging, or both was performed every 3 months for the first 2 years and then every 6 months until disease recurrence or trial completion.

As of the data cut-off, the combination of dabrafenib and trametinib reduced the risk of disease recurrence or death by $53 \%$ compared with placebo (hazard ratio [HR], 0.47; $P<.001)$. Median RFS was not yet reached in the combination arm, compared with 16.6 months in the placebo arm. The RFS benefit was observed across all prespecified

\section{What's new, what's important}

The expanded approval of the dabrafenib-trametinib combination for BRAF-mutant melanoma after complete resection is a welcome option for these patients who often face recurrence. The approval was based on data from the COMBI-AD trial in which 870 patients with stage III melanoma and BRAF V600E/K mutations and lymph-node involvement were randomised either to dabrafenib $150 \mathrm{mg}$ twice daily in combination with trametinib $2 \mathrm{mg}$ once daily, or to 2 matched placebos. Randomization was stratified according to BRAF mutation status and disease stage.

The primary endpoint was RFS, and secondary endpoints included OS, DMFS, FFR, and safety. As of the data cut-off, the dabrafenib-trametinib combination reduced the risk of disease recurrence or death by $53 \%$ compared with placebo (HR, 0.47 ; $P<.001)$. Median RFS was not yet reached in the combination arm (placebo: 16.6 months). The RFS benefit was observed across all prespecified subgroups, and the combination was also found to improve OS, DMFS, and FFR. The most common AEs included pyrexia, fatigue, nausea, and rash, among others.

The prescribing information for the 2 drugs has warnings about their combined use, including the need to confirm BRAF status before starting therapy, new primary malignancies, hemorrhage, cardiomyopathy, uveitis, febrile reactions, skin toxicity, VTE, ocular toxicities, and embryofetal toxicity, some of which can lead to treatment discontinuation. Both drugs can cause fetal harm and patients should be warned of this risk.

- Jame Abraham, MD, FACP (abrahaj5@ccf.org)

subgroups, and the combination was also found to improve OS, DMFS, and FFR.

The most common adverse events (AEs) included pyrexia, fatigue, nausea, rash, vomiting, diarrhea, chills, and myalgia. Overall, $97 \%$ of patients experienced an $\mathrm{AE}, 41 \%$ experienced a grade $3 / 4 \mathrm{AE}$, and $26 \%$ had an $\mathrm{AE}$ that led to treatment discontinuation. In patients treated with placebo, those numbers were $88 \%, 14 \%$, and $3 \%$, respectively.

The separate prescribing information for dabrafenib and trametinib detail warnings and precautions relating to their combined use, including the need to confirm BRAF status before starting therapy (because use in $B R A F$ wildtype tumors can promote tumor cell proliferation), new primary malignancies, hemorrhage, cardiomyopathy, uveitis, serious 


\section{Mechanism of action: dabrafenib and trametinib}

Double hit to a key cancer signaling pathway. The mitogen-activated protein kinase (MAPK) pathway is a key signaling cascade that transmits cell surface stimuli into the nucleus to regulate gene transcription and generate a cellular response. Given its central role in a number of vital cellular processes such as proliferation, growth, and survival, it is not surprising to find that this pathway is frequently dysregulated across tumor types.

This is exemplified by melanoma, in which the most frequently observed recurrent mutations occur in this pathway. In particular, one of the central kinases, BRAF, is mutated in half of all cases, and mutations in a second kinase, MEK, occur in just under $10 \%$ of cases. The most commonly observed BRAF mutations are the $\mathrm{V} 600 \mathrm{E}$ and $\mathrm{V} 600 \mathrm{~K}$ mutations, which occur in $70 \%-95 \%$ and $5 \%-30 \%$ of cases, respectively.

Understandably, the development of targeted therapies for the treatment of metastatic melanoma have focused on the BRAF and MEK proteins. Dabrafenib is a selective, ATP competitive inhibitor of the BRAF V600E mutant protein and trametinib is a selective ATP noncompetitive inhibitor of the MEK $1 / 2$ proteins. Both have shown considerable promise for the treatment of patients with metastatic melanoma that displays $B R A F$ mutations and are approved as single agents for this indication.

However, only a proportion of patients respond to these drugs and, among those who do, resistance typically fuels treatment failure in less than a year. Because activation of other proteins within the MAPK cascade can offer a potential escape route for cancer cells treated with 1 targeted drug, double blockade of the pathway at 2 different points can prove more effective by blocking this route.

The combination of dabrafenib and trametinib has already borne out this hypothesis and is approved for the treatment of metastatic disease. The latest approval demonstrates that the combination is also effective earlier on in the course of the disease, in the adjuvant treatment of patients with melanoma after surgical resection.
RTKs

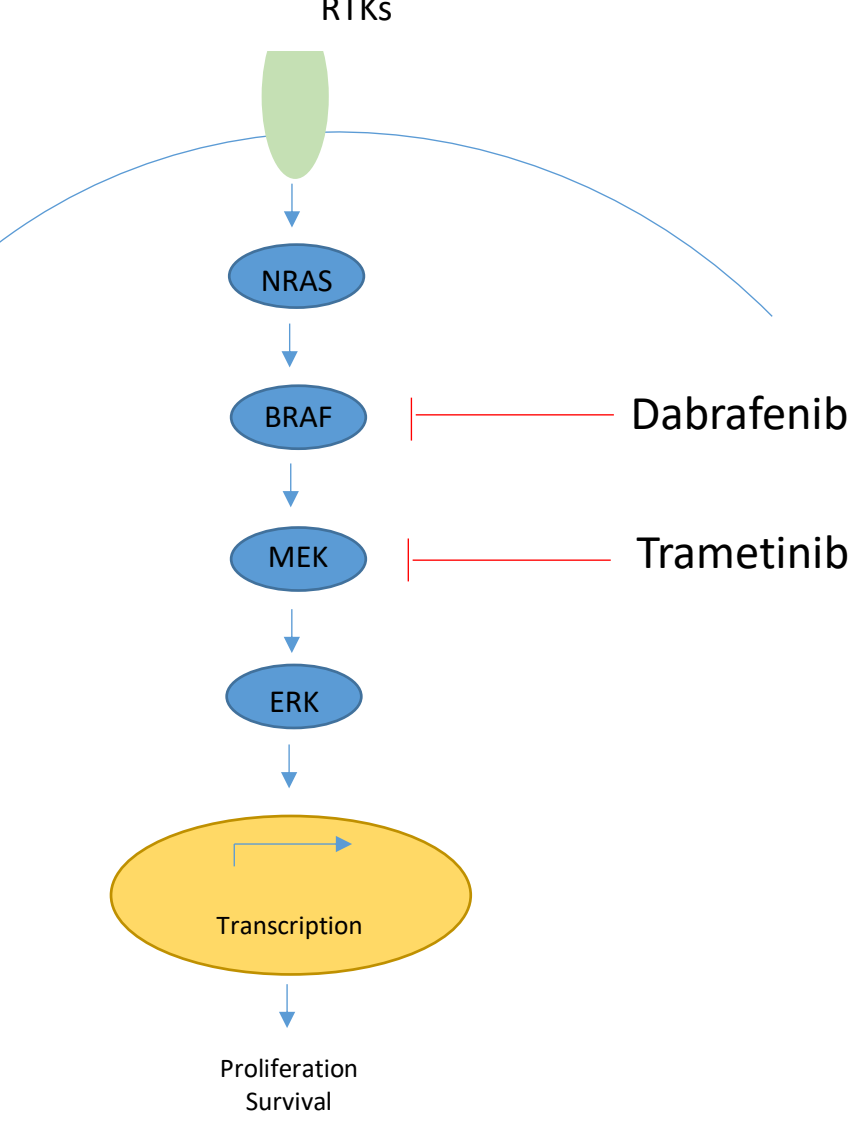

BRAF and MEK, the targets of dabrafenib and trametinib, respectively, are 2 components of the mitogen-activated protein kinase (MAPK) pathway, which is activated by upstream receptor tyrosine kinases (RTKs). Combination therapy with dabrafenib and trametinib serves a double hit to the MAPK pathway, which is frequently upregulated in cancers, including melanoma, and helps to prevent the development of resistance that can arise after monotherapy. Figure generated by Jane de Lartigue. febrile reactions, serious skin toxicity, hyperglycemia, glucose6-phosphate dehydrogenase (G6PD) deficiency, colitis and gastrointestinal perforation, venous thromboembolism, ocular toxicities, interstitial lung disease, and embryofetal toxicity.

Dermatologic evaluations should be completed before starting therapy, every 2 months during and for up to 6 months after completion of therapy, and patients should be monitored closely for the signs and symptoms of noncutaneous primary malignancies. Treatment should be discontinued for all grade 4 hemorrhagic events and for any grade 3 events that do not improve, and withheld for grade 3 events until they resolve, at which point treatment can be resumed at the next lowest dose as described in the prescribing information.

Left ventricular ejection fraction (LVEF) values should be assessed before initiating therapy, after 1 month, and then at intervals of 2-3 months. Treatment should be withheld for up to 4 weeks if absolute LVEF values decrease by $10 \%$ and are less than the lower limit of normal (LLN) and it should be permanently discontinued for symptomatic cardiomyopathy or persistent, asymptomatic left ventricular dysfunction of $>20 \%$ from baseline that is below LLN and does not resolve within 4 weeks.

Treatment should be withheld for fevers higher than 
$104^{\circ} \mathrm{F}$ or for serious febrile reactions or fever accompanied by hypotension, rigors or chills, dehydration, or renal failure. Serum creatinine levels should be monitored, along with other evidence of renal function, during, and after severe pyrexia. Antipyretics should be administered as secondary prophylaxis when treatment is resumed if the patient had previous episodes of severe febrile reaction or if fever was associated with complications. Corticosteroids should be administered for at least 5 days for second or subsequent pyrexia if the body temperature dose not return to baseline within 3 days of fever onset or for pyrexia associated with complications and no evidence of active infection.

Treatment should also be withheld for intolerable or severe skin toxicity and resumed at a lower dose as per guidelines in patients who improve or recover within 3 weeks. Serum glucose levels should be monitored at the start of treatment and as clinically appropriate in patients with pre-existing diabetes or hyperglycemia. Patients with G6PD deficiency should be monitored closely for signs of hemolytic anemia.

Patients should be monitored closely for signs and symp-

\section{References}

1. US Food and Drug Administration Website. FDA approves dabrafenib plus trametinib for adjuvant treatment of melanoma with BRAF V600E or V600K mutations. https://www.fda.gov/drugs/ informationondrugs/approveddrugs/ucm606165.htm. Last updated April 30, 2018. Accessed October 6, 2018.

2. Long GV, Hauschild A, Santinami M, et al. Adjuvant dabrafenib plus trametinib in stage III BRAF-mutated melanoma. N Engl J Med. 2017;377:1913-1823. toms of colitis and gastrointestinal perforation and should be advised to immediately seek medical care if they develop symptoms of deep vein thrombosis (DVT) or pulmonary embolism (PE). Treatment should be permanently discontinued for life-threatening PE, or withheld for uncomplicated DVT and PE for up to 3 weeks and then resumed at a lower dose if the patient improves.

Ophthalmological evaluations should be performed periodically and within 24 hours of patient-reported loss of vision or other visual disturbances. Treatment should be permanently discontinued in patients with documented retinal vein occlusion and withheld for retinal pigment epithelial detachment. Treatment should also be withheld in patients presenting with new or progressive pulmonary symptoms and findings and permanently discontinued for treatment-related interstitial lung disease or pneumonitis.

Both dabrafenib and trametinib can cause fetal harm and patients should be warned of this risk and the need for adequate contraceptive measures. Dabrafenib and trametinib are marketed as Tafinlar and Mekinist by Novartis.

3. Tafinlar (dabrafenib) capsules, for oral use. Prescribing information. Novartis. https://www.pharma.us.novartis.com/sites/www.pharma. us.novartis.com/files/tafinlar.pdf. May 2018. Accessed October 6, 2018.

4. Mekinist (trametinib) tablets, for oral use. Prescribing information. Novartis. https://www.pharma.us.novartis.com/sites/www.pharma. us.novartis.com/files/mekinist.pdf. May 2018. Accessed October 6th, 2018. 